a Departamento de Ciências Naturais, Universidade Federal de São João del Rei, Campus Dom Bosco, Praça Dom Helvécio 74, Fábricas, CEP 36301-160, São João del Rei-MG, Brasil.

*E-mail: arnaldocsp@yahoo.com.br

Recebido em: 13 de Fevereiro de 2021

Aceito em: 4 de Agosto de 2021

Publicado em: 3 de Fevereiro de 2022

\section{Synthesis, Structural and Electrochemical Characterization of Nanomaterials Aiming Its Application in Electrochemical Sensor Development}

\author{
Síntese e Caracterização Estrutural e Eletroquímica de Nanomateriais \\ Visando Sua Aplicação no Desenvolvimento de Sensores Eletroquímicos
}

Laís Sales Portoa, Arnaldo César Pereira ${ }^{a, *}$

\begin{abstract}
In this work the effect of nanocomposite based on functionalized Carbon Nanotubes decorated with Silver Nanoparticles (CNTf-AgNP) and Gold Nanoparticles (CNTf-AuNP) as a modifier of Pyrolytic Graphite Electrode (PGE) surface was evaluated. By combining these two classes of nanomaterials, it is expected to obtain a synergistic effect that adds the high catalytic power of Metallic Nanoparticles (NPs) with the Carbon Nanotubes (CNT) excellent electrical conductivity, enabling an improvement in kinetics of redox processes, expanding the electrochemical signal and increasing the sensor sensitivity. The materials were synthesized and characterized by UV-Vis Spectroscopy, Fourier Transform Infrared Spectroscopy (FT-IR), Scanning Electron Microscopy (SEM) and X-Ray Diffraction (XRD). Electrochemical characterization was performed by Electrochemical Impedance Spectroscopy (EIS) and Cyclic Voltammetry (CV). The results confirmed the synthesis of CNTf-AgNP and CNTf-AuNP nanocomposites. Furthermore, CNTf-AgNP significantly enhanced the electroactive surface area of PGE, decreased the resistance to electron transfer and considerably increased the electron transfer rate, with current gain approximately $300 \%$ larger than the electrode without any modification. Therefore, the development of an electrochemical sensor obtained by a fast and simple solvent evaporation process was carried out effectively, with great potential and viability to be applied on different compounds of clinical, industrial and environmental interest determination.
\end{abstract}

Keywords: Electrochemical sensor; characterization; carbon nanotubes; silver nanoparticles; gold nanoparticles

\section{Introduction}

Long before receiving this denomination, Nanoscience and Nanotechnology $(\mathrm{N} \& \mathrm{~N})$ was already present in ideas and concepts studied by several research groups around the world, especially those with studies aimed at miniaturizing electronic systems and nanoparticulate systems. ${ }^{1}$ The advancement of research and the discovery of new materials with particles on nanometers order, led N\&N to consolidate itself as a new area of knowledge at the end of the 20th century. Nowadays, $\mathrm{N} \& \mathrm{~N}$ is one of the fastest growing areas due to its great interdisciplinarity and potential for application in different sectors of human activity such as chemistry, physics, biology, engineering and medicine. ${ }^{2}$

Nanomaterials, by definition, must have at least one of dimensions at nanoscale and present different properties from those observed for the same material at macroscopic scale, knowing that materials properties (optical, magnetic, electrical, catalytic, etc.) depends not only on their composition and structure, but also on their shape and size, also known as critical size. Thus, when material's particles are below the critical size, its properties change, which makes it possible to prepare new materials just controlling the size and shape of its particles. ${ }^{3}$

In recent years, the modification of electrochemical sensors with nanomaterials has attracted attention and shown to be extremely promising due to benefits as low cost, reagent reduction, fast response, portability, increased sensitivities, reduced overpotentials, resistance to surface fouling and several possibilities for applying these devices such in fundamental biological research, health monitoring, clinical diagnostics, pharmaceutical analyzes, quality control, food safety and environmental monitoring. ${ }^{3-5}$

Two of the most expressive examples of nanomaterials class, especially in electroanalytical applications, are Carbon Nanomaterials (CNs) and Metal Nanoparticles (NPs). Carbon Nanotubes (CNT) are one of the most representative CNs that has attracted enormous interest due to its excellent mechanical, physical and chemical properties, result of its unique structure. ${ }^{6}$ 
The CNT have several attractive properties for the development of electrochemical sensors, such as: excellent thermal and electrical conductivity; unique electrocatalytic activity; extraordinary chemical stability; high tensile strength; high electron transfer speed; axial structure with large surface area and small diameter. All these unique properties of CNT make it as an ideal and efficient matrix for dispersing and stabilizing NPs, allowing its use as a template for preparing CNT/NPs nanohybrids, further improving the electron transfer between analyte and electrode. ${ }^{6-9}$

Over last twenty years the construction of electrochemical sensors modified with CNT decorated with noble metals nanoparticles (Ag, Au, Pt and Pd) has received considerable attention due to significant advantages of using NPs such as easy synthesis, easy handling and surface functionalization, efficient catalytic effect in electrochemical reactions, improving electron transfer process. ${ }^{9}$

Moreover, formation of nanocomposite from NPs and CNT causes a synergistic effect that adds the properties of the two nano-sized composites, reduces overvoltage, improves the kinetics of electrode's redox processes, has a progressive effect on the mass transfer and increases the sensitivity and selectivity of the base electrochemical sensor, which demonstrates the great potential of using these nanocomposites to modify electrochemical sensors for various applications. ${ }^{9-11}$

In the present work, AgNP and AuNP were chosen because its important characteristics such high stability, excellent conductivity, effective catalytic behavior and excellent biocompatibility. ${ }^{5,10}$ Therefore, this work aims the development of an electrochemical sensor modified by nanocomposite based on functionalized Carbon Nanotubes decorated with Silver nanoparticles (CNTfAgNP) and Gold Nanoparticles (CNTf-AuNP) obtained by a fast and simple solvent evaporation process for future application on different compounds of clinical, industrial and environmental interest determination.

\section{Experimental}

\subsection{Reagents and Solutions}

Multi-walled Carbon Nanotubes (MWCNT), Nitric Acid $\left(\mathrm{HNO}_{3}\right)$, Acetic Acid $\left(\mathrm{CH}_{3} \mathrm{COOH}\right)$, Boric Acid $\left(\mathrm{H}_{3} \mathrm{BO}_{3}\right)$, were obtained from Synth ${ }^{\circledast}$. Silver Nitrate $\left(\mathrm{AgNO}_{3}\right)$, Phosphoric Acid $\left(\mathrm{H}_{3} \mathrm{PO}_{4}\right)$, Sodium Borohydride $\left(\mathrm{NaBH}_{4}\right)$, Sodium Tetrachloroaurate $\left(\mathrm{NaAuCl}_{4}\right)$, and Sodium Hydroxide $(\mathrm{NaOH})$ were purchased from Sigma-Aldrich ${ }^{\circledR}$. Sulfuric Acid $\left(\mathrm{H}_{2} \mathrm{SO}_{4}\right)$ is from Dinâmica ${ }^{\circledR}$ and Sodium Citrate $\left(\mathrm{Na}_{3} \mathrm{C}_{6} \mathrm{H}_{5} \mathrm{O}_{7}\right)$ from Cinética ${ }^{\circledR}$.

All chemical reagents used were in analytical purity and solutions were prepared in distilled and deionized water by the Millipore Milli-Q system (resistivity $>18 \mathrm{M} \Omega \mathrm{cm}^{-1}$, $\left.25^{\circ} \mathrm{C}\right)$.

\subsection{Carbon Nanotubes Functionalization}

In order to occur an effective deposition of NPs onto CNT surface, it is necessary to first perform an oxidative treatment to introduce oxygen-containing functionalities such as hydroxyl and carboxyl groups at CNT walls to which the NPS can bind. Often this process involves harsh treatments of CNT with concentrated mixtures of strong oxidizing acids, such as sulfuric and nitric acid. ${ }^{12}$

Therefore, $0.5 \mathrm{~g}$ of commercial MWCNT were weighed. Then, in a erlenmeyer, $250 \mathrm{~mL}$ of $\mathrm{H}_{2} \mathrm{SO}_{4}(96 \% \mathrm{v} / \mathrm{v})$ and $\mathrm{HNO}_{3}(65 \% \mathrm{v} / \mathrm{v})$ in $3: 1 \mathrm{v} / \mathrm{v}$ ratio were slowly added. This mixture was taken to ultrasound bath for $2 \mathrm{~h}$. After that, the mixture was kept at rest for $20 \mathrm{~h}$. After completing the reaction, the product was centrifuged $(4000 \mathrm{rpm})$ and washed by doubly distilled water several times until a pH close to neutral $(\mathrm{pH} \mathrm{7,0)}$ was obtained. Finally, the solid obtained was vacuum filtered and dried in oven at $110^{\circ} \mathrm{C}$ for approximately $4 \mathrm{~h}$ to obtain the Functionalized Carbon Nanotubes (CNTf).

\subsection{Silver Nanoparticles Synthesis}

$\mathrm{AgNPs}$ were prepared by chemical reduction of $\mathrm{AgNO}_{3}$ by $\mathrm{NaBH}_{4}$ in the presence of sodium citrate to stabilize the nanoparticles. Thereunto, two equimolar solutions of $\mathrm{AgNO}_{3}$ (silver nanoparticles precursor) and $\mathrm{Na}_{3} \mathrm{C}_{6} \mathrm{H}_{5} \mathrm{O}_{7}$ (stabilizing agent) at $0.67 \mathrm{mmol} \mathrm{L}^{-1}$ were prepared. Then, $45 \mathrm{~mL}$ of each solution was mixed and kept in ice bath for $1 \mathrm{~h}$. After that, $10 \mathrm{~mL}$ of $27 \mathrm{mmol} \mathrm{L}^{-1} \mathrm{NaBH}_{4}$ (solution prepared and previously stored in the refrigerator for 1 hour) was added. In this reaction, $\mathrm{NaBH}_{4}$ acts as reducing agent for $\mathrm{Ag}{ }^{+}$ions to $\mathrm{Ag}^{0} .{ }^{13}$

After $\mathrm{NaBH}_{4}$ addition, the solution color changed from transparent to light yellow, which is characteristic of silver nanoparticles colloidal dispersion formation. ${ }^{14}$ Finally, the solution was stirred for another $2 \mathrm{~h}$ in ice bath and stored at the refrigerator in amber bottle.

The choice of using $\mathrm{NaBH}_{4}$, considered a strong reducing agent, sodium citrate for stabilization and carrying out the reaction at low temperature, aimed at obtaining nanoparticles with reduced size. Changing any of these factors can result in the clustering of the nucleation centers, forming $\mathrm{Ag}^{0}$ particles with diameters larger than the nanometric scale.

\subsection{Gold Nanoparticles Synthesis}

Chemical reduction by sodium citrate is the most conventional method used to prepare monodispersed gold nanoparticles since 1953. ${ }^{15}$ Unlike the AgNPs synthesis, in the AuNPs synthesis process, citrate is used as reducing and stabilizing agent simultaneously, resulting in a uniform and stable AuNPs colloidal dispersion.

Thus, for AuNPs synthesis, $0.125 \mathrm{~mol} \mathrm{~L}^{-1} \mathrm{NaAuCl}_{4}$ solution and $0.04 \mathrm{~mol} \mathrm{~L}^{-1}$ sodium citrate solution was 
prepared. Then, $420 \mu \mathrm{L}$ of $\mathrm{NaAuCl}_{4}$ was added to $99.6 \mathrm{~mL}$ of water. The reaction mixture was stirred and heated until it reached $90{ }^{\circ} \mathrm{C}$. Upon reaching this temperature, $5 \mathrm{~mL}$ of sodium citrate was added. The reaction proceeded for 20 minutes under constant agitation and temperature. After this stage, the solution color changed from transparent to red. The mixture was gradually cooled to room temperature and stored in the refrigerator in an amber bottle. The solution color change is an indication that the gold nanoparticles colloidal dispersion formation occurred effectively. ${ }^{16}$

\subsection{Apparatus}

Voltammetric experiments were performed using a Metrohm potentiostat/galvanostat model PGSTAT101, with Ecochemie's FRA32M impedance module, coupled to a computer containing the Nova 2.1 software. A conventional three electrode system was used with a basal plane Pyrolytic Graphite as working electrode (PGE, unmodified or modified, $d=6 \mathrm{~mm}$ ), an $\mathrm{Ag} / \mathrm{AgCl}$ reference electrode $\left(3 \mathrm{~mol} \mathrm{~L}^{-1} \mathrm{KCl}\right)$ and a Pt wire counter electrode.

Electrochemical impedance spectroscopy (EIS) measurements were performed with $5.0 \mathrm{mmol} \mathrm{L}^{-1}\left[\mathrm{Fe}(\mathrm{CN})_{6}\right]^{3-1}$ $\left[\mathrm{Fe}(\mathrm{CN})_{6}\right]^{4-}(1: 1)$ in $0.1 \mathrm{~mol} \mathrm{~L}^{-1} \mathrm{KCl}$ solution $\mathrm{pH} 7.0$. The frequency range was between $100 \mathrm{KHz}$ and $0.1 \mathrm{~Hz}$, with application of $0.249 \mathrm{~V}$ potential (DC) and $0.01 \mathrm{~V}$ amplitude (AC). Solutions were deaerated by bubbling nitrogen prior to the experiments. All experiments were performed at $25 \pm 1{ }^{\circ} \mathrm{C}$.

UV-Vis spectra were recorded by a UV-2550 spectrophotometer from Shimadzu. The AgNPs and AuNPs samples were diluted in deionized water by a known factor and placed in a $1 \mathrm{~cm}$ optical path quartz cuvette. The spectra were recorded in a scanning range between 300 and 700 $\mathrm{nm}$. At each $1 \mathrm{~nm}$ wavelength interval, absorbance values were subtracted from the cuvette containing deionized water, as a baseline. For NPs, the region of interest of the electromagnetic spectra is close to the transition from the visible to the ultraviolet.

Aimed to obtain information about the morphology and size of the nanoparticles, Scanning Electron Microscopy (SEM) images were taking by using an JEOL instrument JSM7500F model, operating at $200 \mathrm{kV}$. This analysis was carried out at Universidade Estadual Paulista (UNESP), Araraquara - SP, by Profa. Dra. Maria Pilar Taboada Sotomayor, collaborator of Prof. Dr. Arnaldo César Pereira.

$\mathrm{X}$-Ray Diffraction (XRD) was also taken using a Shimadzu diffractometer (XRD 6000 model) using $\mathrm{CuK} \alpha$ radiation with a wavelength $(\lambda)$ of $0.154060 \mathrm{~nm}(40 \mathrm{kV}$, $30 \mathrm{~mA}$ ) and a scanning speed of $2 \mathrm{~min}^{-1}$, covering the $2 \theta$ range from 10 to $80^{\circ}$. Fourier Transform Infrared (FT-IR) spectra was recorded from 4000 to $400 \mathrm{~cm}^{-1}$ with $2 \mathrm{~cm}^{-1}$ of resolution and 64 number of scans, by an Agilent Fourier Transform Infrared Spectrometer Cary 630 FT-IR model. All samples were dispersed in $\mathrm{KBr}$ disks by hydraulic press. Infrared spectra and X-ray diffractogram were performed at
Universidade Federal dos Vales do Jequitinhonha e Mucuri (UFVJM), Diamantina - MG, by Prof. Dr. Lucas Franco Ferreira who is a collaborator of Prof. Dr. Arnaldo César Pereira.

\subsection{Electrode Modification}

Before the stage of modifying the working electrode surface, PGE was polished to with $0.05 \mu \mathrm{m}$ alumina powder until a mirror-shiny surface was obtained. Then, the electrode was cleaned with ethanol and a bath ultrasonic cleaner was used to remove the rest of particles from the electrode surface.

For PGE modification, $4 \mathrm{mg}$ of CNTf were weighed and added, separately, in $2 \mathrm{ml}$ of AgNPs and AuNPs colloidal dispersions. Thereafter, these dispersions were taken to the bath ultrasonic for 30 minutes. Subsequently, two chemically modified electrodes were prepared by adding a $10 \mu \mathrm{L}$ aliquot of each dispersion on PGE surface. Finally, the modified electrode was dried in the oven at $60^{\circ} \mathrm{C}$ for 20 minutes, making it possible to obtain PGE / CNTf-AgNP and PGE / CNTf-AuNP sensors.

\section{Results and Discussion}

\subsection{AgNPs and AuNPs UV-Vis Spectroscopy Characterization}

$\mathrm{UV}$-Vis spectroscopy was chosen because it is the most used method for NPs characterization, since the interaction of NPs with electromagnetic radiation is more intense in the region close to the transition from the visible to the ultraviolet of the electromagnetic spectra, providing important information about the condition of the colloidal dispersion. ${ }^{17}$ The incident electromagnetic radiation frequency capable of resonating with the NPs electron oscillation frequency varies depending on the shape, size and organization of the nanoparticles. Thus, for nanoparticles of the same nature it is possible to observe different resonances frequencies through the variation of any of these properties, allowing the characterization and distinction of different NPs through UV-Vis absorption spectra analysis. ${ }^{17-19}$

Therefore, UV-vis spectra were acquired in order to observe the characteristic Surface Plasmon Resonance (SPR) bands of AgNPs and AuNPs. The SPR effect occurs when the NPs electron oscillation frequency resonates with the incident electromagnetic radiation frequency. For AgNPs and AuNPs, this occurs in the visible region, which causes colloidal dispersions to show a characteristic color. ${ }^{18,19}$

As shown in Figure 1, AgNPs and AuNPs colloidal dispersions showed a yellowish color and deep red color, respectively, with no precipitate, indicating the achievement of stable nanoparticles and suggesting that both NPs were successfully synthesized. Their characteristic SPR bands were observed with absorption maxima $(\lambda \max )$ at $\sim 390 \mathrm{~nm}$ 

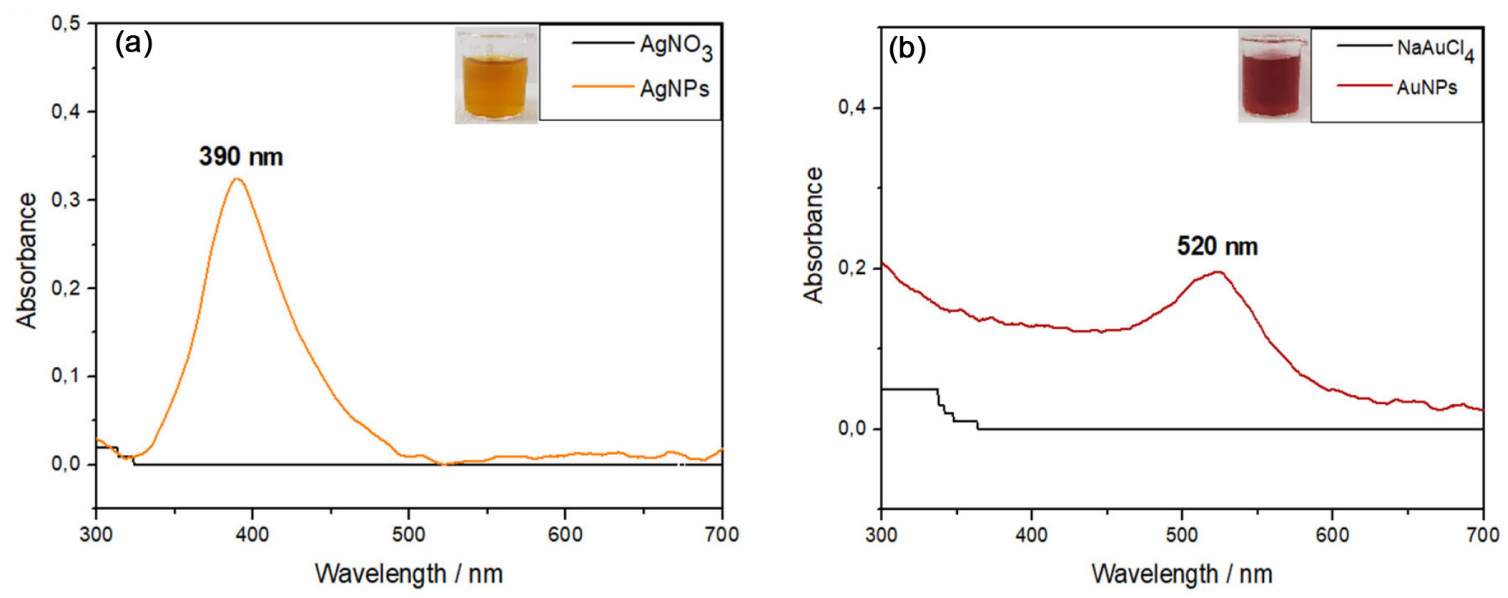

Figure 1. UV-vis spectra of (a) AgNPs (b) AuNPs

for AgNPs (Figure 1a) and $\lambda \max \sim 520 \mathrm{~nm}$ for AuNPs (Figure 1b).

From UV-Visible spectroscopic studies, it is also inferred that the decrease observed in absorbance and the widening of AuNPs band (Figure 1b) indicates a greater aggregation when compared to AgNPs dispersion, resulting in an increase in the average diameter for AuNPs, between 15 and $20 \mathrm{~nm}$, compared to AgNPs with an expected average diameter between 10 and $15 \mathrm{~nm} \cdot{ }^{16-20}$

\subsection{Functionalized Carbon Nanotubes FT-IR Characterization}

FT-IR analysis was performed to characterize functional groups on the carbon nanotubes structure after treatment with strong oxidizing acids. The obtained spectra from 4000 to $400 \mathrm{~cm}^{-1}$ of pristine MWCNT and functionalized CNT are illustrated in Figure 2.

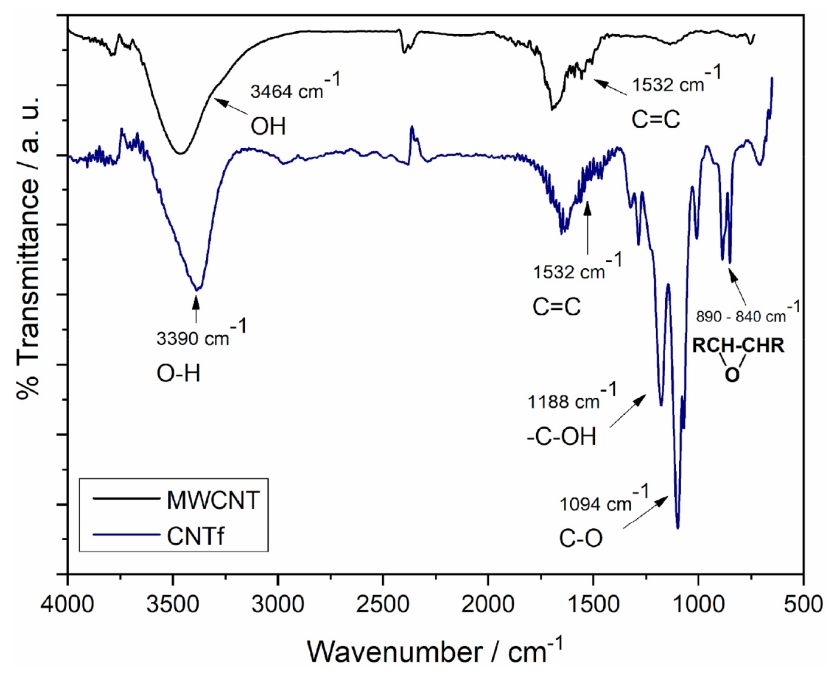

Figure 2. FT-IR spectra of commercial MWCNT and functionalized CNT

The FT-IR spectra confirmed the integrity of hexagonal structure of MWCNT by the presence of peak at $1567-1532 \mathrm{~cm}^{-1}$ that occur due to the existence of carbon double bonding $(\mathrm{C}=\mathrm{C})$ and are related to elongation and contraction movement of carbon nanotubes tubular structure. ${ }^{21}$ In addition, it was noticeable an intense peak at $3460 \mathrm{~cm}^{-1}$ which may be associated with stretching hydroxyls $(\mathrm{O}-\mathrm{H})$ in the carboxyl groups or possible adsorbed water molecules. ${ }^{22}$

The spectra of CNTf also shows a peak attributed to the stretching of the hydroxyl $\mathrm{O}-\mathrm{H}$ bond, however in greater intensity and with a displacement of the band to $3390 \mathrm{~cm}^{-1}$. Besides that, it was observed the occurrence of an intense peak at $1188 \mathrm{~cm}^{-1}$, corresponding to the $-\mathrm{C}-\mathrm{O}-\mathrm{H}$ deformation of alcohols, and a peak at $1094 \mathrm{~cm}^{-1}$ related to the $-\mathrm{C}-\mathrm{O}$ stretching of ethers. Appearance of peak at $890-840 \mathrm{~cm}^{-1}$ assigns symmetrical and asymmetric deformation of epoxides on CNTf structure, which are absent at MWCNT spectra. ${ }^{23}$

This fact can be explained due to the treatment with sulfuric acid and nitric acid which MWCNT were submitted. Usage of both strong oxidizing acids in the purification process causes partial oxidization of carbon and, consequently, increases the number of functional groups whereas the acidic solution attacks double bonding between the carbon atoms..$^{21,22}$ Therefore, through FT-IR analysis, it was possible to confirm the increase of functional groups in MWCNT structure, proving the formation of CNTf.

\subsection{Nanocomposite X-Ray Diffraction Characterization}

The characterization of the structure and chemical composition of the CNTf-AgNP and CNTf-AuNP nanocomposites was carried out through XRD analysis. The XRD diffraction patterns for each nanocomposite are shown in Figure 3.

The XRD patterns clearly show a typical characteristic peak that confirm the immobilization of AgNPs and AuNPs on CNTf structure. The XRD pattern for CNTf-AgNP (Figure 3a) exhibited peaks at $2 \theta$ values of $38.26^{\circ}, 44.37^{\circ}$, $64.68^{\circ}$ and $77.63^{\circ}$, corresponding to (111), (200), (220) and (311), crystal planes of metallic Ag respectively, related to 

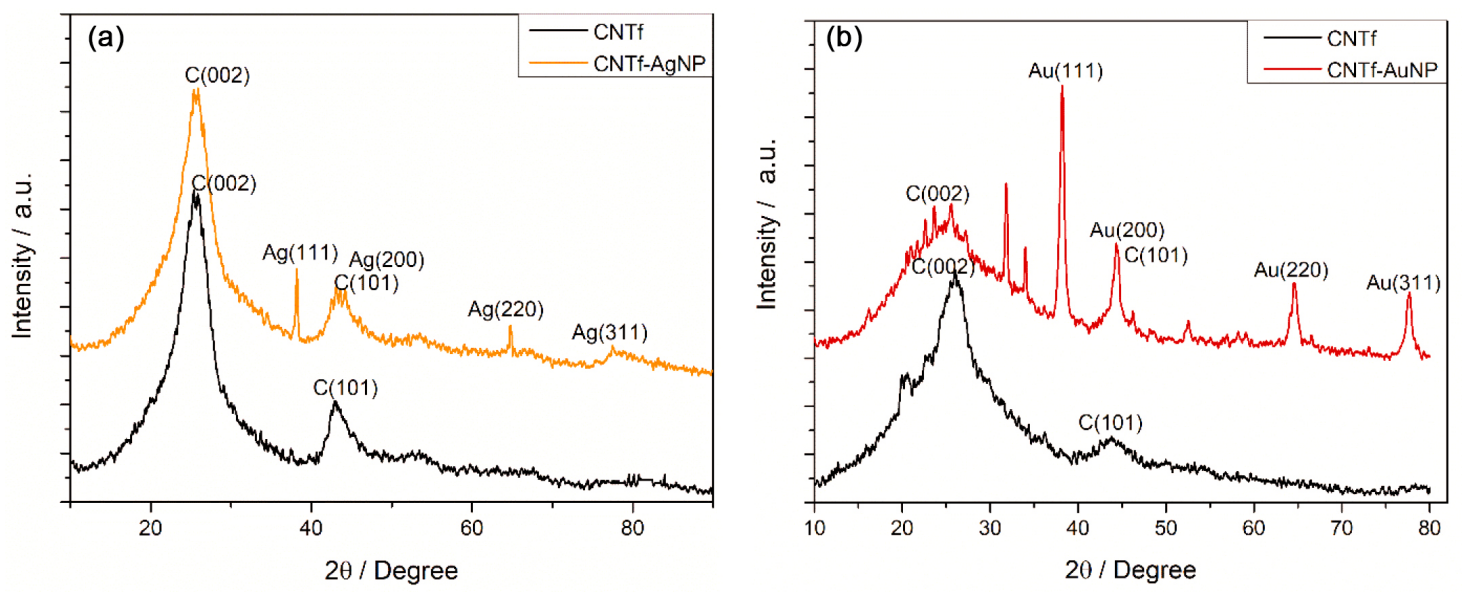

Figure 3. XRD patterns of (a) CNTf-AgNP and (b) CNTf-AuNP

Face - Centered Cubic (FCC) lattice of AgNPs. ${ }^{24}$ Also, the peaks observed at $2 \theta$ of $25.86^{\circ}, 43.12^{\circ}$, referring to plans $\mathrm{C}$ (002) and C (101), related to the graphitized nature of CNTf, remained even after AgNPs immobilization, indicating that CNTf structural organization did not change during the immobilization process. ${ }^{9}, 17$

Similarly, the CNTf-AuNP nanocomposite formation was confirmed (Figure $3 \mathrm{~b}$ ) by the presence of $2 \theta$ peaks at $38.20^{\circ}, 44.42^{\circ}, 64.67^{\circ}$ and $77.74^{\circ}$, referring to Bragg's reflections from the planes (111), (200), (220) and (311), of the FCC lattice of AuNPs. ${ }^{25}$ The peaks related to C (002) and C (101) planes of the CNTf structure, also remained after AuNPs immobilization with $2 \theta$ diffraction angles at $26.09^{\circ}$ and $43.80^{\circ}$, respectively.

Furthermore, the size of each metallic nanoparticle was estimated by the Scherrer equation (equation 1 ):

$$
\mathrm{L}=\frac{0.9 \lambda}{\beta \cos \theta}
$$

The definitions are as follows: $\mathrm{L}$ is a measure of the particle size in the direction perpendicular to the reflecting plane (which will be calculated), $\lambda$ is the wavelength of the applied $\mathrm{X}$-rays, $\beta$ is the full width at half-maximum (FWHM) of the diffraction peak in radians and $\theta$ is Bragg's angle. The results of the average sizes of AgNPs and AuNPs crystals estimated by the Scherrer equation are shown in Table 1.

Although the Scherrer equation makes it possible to obtain a rough estimate of nanoparticle size, this method neglects the importance of the contribution of the deviations caused by the diffraction equipment. Therefore, the $\beta$ parameter needs to be corrected to eliminate the so-called instrumental effects. ${ }^{26}$

A simple method to separate the contribution of the enlargement caused by the equipment from the actual particle size in the XRD patterns is the Williamson-Hall plotting. Thus, the microstrain $(\varepsilon)$ and the particle size $(\mathrm{L})$ of AgNPs and AuNPs were also calculated by the WilliamsonHall (W-H) equation (equation 2): ${ }^{26}$

$$
\frac{\beta \cos \theta}{\lambda}=\frac{K}{L}+\frac{4 \varepsilon}{\lambda} \sin \theta
$$

\begin{tabular}{|c|c|c|c|c|c|}
\hline $\begin{array}{l}\text { Crystallographic } \\
\text { direction }\end{array}$ & $\begin{array}{l}\text { Scherrer } \\
\text { Equation }\end{array}$ & $\begin{array}{c}\text { Scherrer equation } \\
\text { (Corrected line } \\
\text { width) }\end{array}$ & $\begin{array}{c}\text { Average Size by } \\
\text { Scherrer equation } \\
\text { (Corrected line } \\
\text { width) }\end{array}$ & \multicolumn{2}{|c|}{ Williamson-Hall plotting } \\
\hline hkl & $\mathbf{L}(\mathbf{n m})$ & $\mathbf{L}(\mathbf{n m})$ & $\underline{\mathbf{L}}(\mathbf{n m})$ & $\mathbf{L}(\mathbf{n m})$ & $\varepsilon$ \\
\hline \multicolumn{6}{|c|}{ AgNP } \\
\hline 111 & 15.02 & 18.44 & 15.74 & 15.52 & 0.005 \\
\hline 200 & 11.21 & 13.35 & & & \\
\hline 220 & 10.95 & 14.06 & & & \\
\hline 311 & 12.17 & 17.09 & & & \\
\hline \multicolumn{6}{|c|}{ AuNP } \\
\hline 111 & 27.12 & 27.18 & 26.70 & 26.47 & 0.349 \\
\hline 200 & 25.24 & 26.30 & & & \\
\hline 220 & 25.41 & 26.50 & & & \\
\hline 311 & 26.71 & 26.82 & & & \\
\hline
\end{tabular}

Table 1. Particle sizes calculated by Scherrer equation, Scherrer equation (corrected line width) and WH plotting 
where $\beta$ is the width of the peak at half the maximum intensity (as the sum of the peak width due to the microstrain and due to particle size), $\theta$ the Bragg diffraction angle, $\lambda$ the $\mathrm{K} \alpha$ radiation $(\sim 0.15406 \mathrm{~nm}), \mathrm{L}$ the particle size and $\varepsilon$ the microstrain.

The microstrain $(\varepsilon)$ is estimated from the slope of the $\mathrm{W}-\mathrm{H}$ line plot and the particle size $(\mathrm{L})$ from the intersection with the vertical axis. ${ }^{26,27}$ Figure 4 shows the W-H plots of both the samples of AgNPs and AuNPs. The results of L and $\varepsilon$ by W-H equation are shown in Table 1 .

As can be seen in Table 1 the average particle size obtained using the corrected line width in the Scherrer equation is $15.74 \mathrm{~nm}$ for AgNPs and $26.70 \mathrm{~nm}$ for AuNPs. The average particle size calculated using the WH plotting has presented very similar values as $15.52 \mathrm{~nm}$ for AgNPs and $26.47 \mathrm{~nm}$ for AuNPs. However, the AuNPs shown higher values of microstrain than AgNPs confirming the expansion of the AuNPs unit cell volume.

Furthermore, these values are in agreement with what was expected after the characterization by UV-Vis spectroscopy (Topic 3.1) and confirm the formation of monodisperse metallic nanoparticles with small diameter well distributed over CNTf surface.

\subsection{Scanning Electron Microscopy}

The morphologies of prepared CNTf decorated with AgNPs and AuNPs were studied by Scanning Electronic

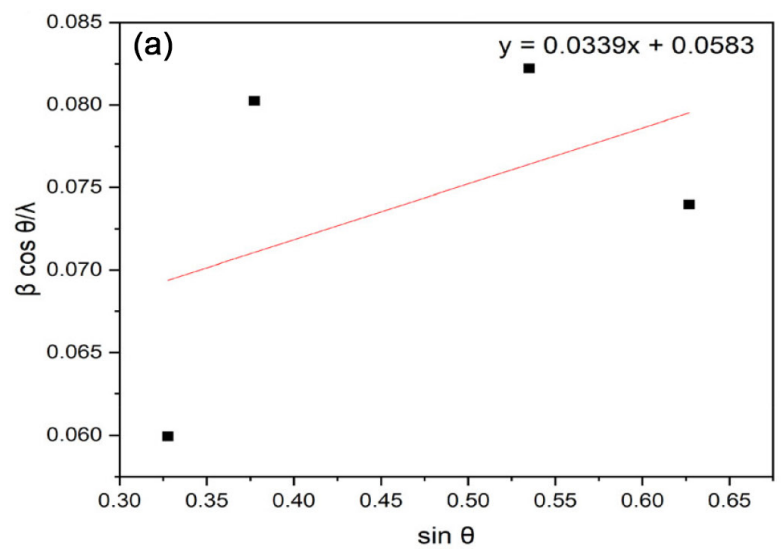

Microscopy (SEM), as illustrated in Figure 5. The representative SEM images of the CNTf-AgNP and CNTf-AuNP demonstrated a homogeneous distribution of nanoparticles covering the entire functionalized carbon nanotubes surface. It can be also observed the presence of a few clusters, showing good dispersion, which means that metal nanoparticles have been successfully adsorbed on CNTf structure. This good dispersion of NPs on CNTf structure creates a constructive network that can stimulate an increase in the electron's transfer speed. ${ }^{17}$

In addition, it can be highlighted that the CNTf's morphology (Figure 5a) remained unchanged after the functionalization and NPs immobilization process. The treatment with strong oxidizing acids did not cause samples decomposition. However, it was possible to observe an increase in open extremities, characterizing the formation of highly reactive sites due to the higher concentration of oxygenated chemical groups.

Therefore, given the results presented, it was possible to characterize the formation of nanocomposites based on CNTfAgNP and CNTf-AuNP which, when applied as chemical modifiers, can increase the conductivity and sensitivity of the base working electrode in electrochemical system.

\subsection{Electrochemical Characterization}

Electrochemical Impedance Spectroscopy (EIS) was employed in order to characterize the interfacial variations

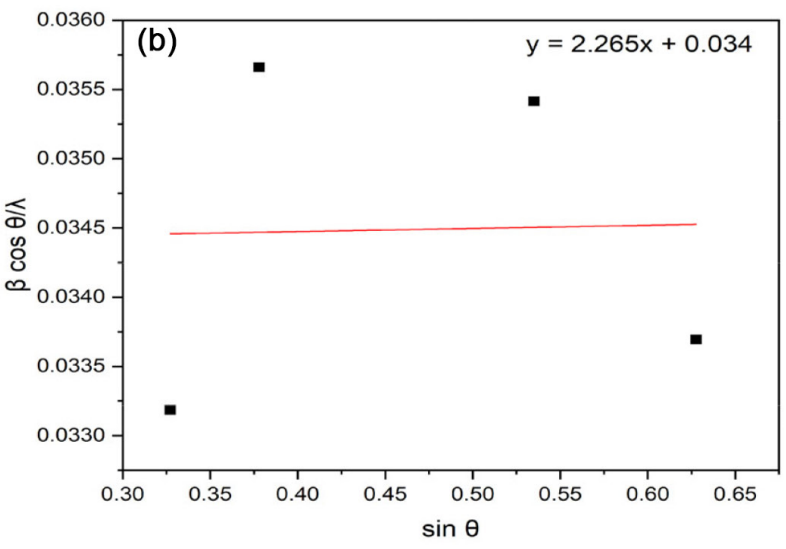

Figure 4. Williamson-Hall plotting to (a) AgNPs and (b) AuNPs
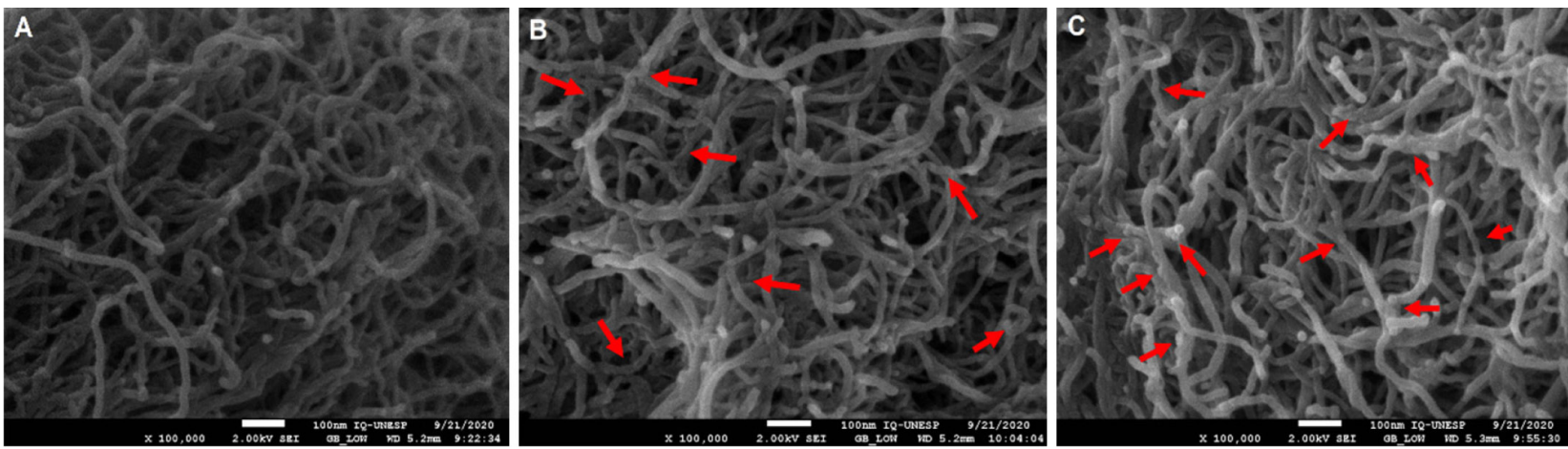

Figure 5. SEM images of (a) CNTf (b) CNTf-AgNP (c) NTCf-AuNP 
of the electrode surface and to investigate the impedance changes based on different electrode configurations. In EIS, the semicircular part at higher frequencies correspond to the electron (charge) transfer resistance (Rct) and linear part at lower frequencies is related to the diffusion process. Thus, the diameter of the semicircle equals Rct represents the difficulty of electron transfer between the solution and the electrode, supplying information of the electrochemical interphase. $^{28}$

Figure 6 shows the Nyquist diagrams for EIS measurements in $\mathrm{K}_{3}\left[\mathrm{Fe}(\mathrm{CN})_{6}\right] / \mathrm{K}_{4}\left[\mathrm{Fe}(\mathrm{CN})_{6}\right]$ and $0.1 \mathrm{~mol} \mathrm{~L}^{-1}$ $\mathrm{KCl}$ for the bare PGE; PGE/CNTf; PGE/ CNTf-AgNP and PGE/CNTf-AuNP. Through the analysis of the diagram it was possible to observe a semicircle with a large diameter for the bare PGE $(249 \mathrm{~K} \Omega$ ). The diameter of this semicircle is reduced to PGE/CNTf (173 $\mathrm{k} \Omega$ ), PGE/CNTf-AuNP $(130 \mathrm{k} \Omega)$ and PGE/CNTf-AgNP $(56 \mathrm{k} \Omega)$, respectively. These results suggest that the surface of the PGE modified with the nanocomposite based on CNTf-AgNP has less resistance to electron transfer between the electrode/ solution interface. The observed improvements, in terms of conductivity, are attributed to the high surface area of CNTf-AgNP nanocomposite, to AgNPs electrocatalytic effect and CNTf excellent conductive properties which, as consequence, decrease Rct and greatly increases the electron-transfer rate.

In addition, the electrochemical response of PGE/AgNP electrode, compared to the bare PGE response, in a $0.1 \mathrm{~mol} \mathrm{~L}^{-1} \mathrm{KCl} \mathrm{pH} 7.0$ solution, was carried out by Cyclic Voltammetry (CV) and the result is illustrated in Figure 7a. From the analysis of the voltammograms it was possible to observe a pair of redox peaks for PGE/AgNP at potentials +0.15 and $0.00 \mathrm{~V}$, related to the redox behavior of AgNPs on the modified electrode surface. ${ }^{29}$ Figure $7 \mathrm{~b}$ shows cyclic voltammograms for bare PGE and PGE/AuNP, in $0.1 \mathrm{~mol} \mathrm{~L}^{-1} \mathrm{KCl}$ solution. A pair of anodic peaks were observed at $+0.15 \mathrm{~V}$ and $+0.98 \mathrm{~V}$, and cathodic peaks at $-0.46 \mathrm{~V}$ and $0.00 \mathrm{~V}$. It can be suggested that the peaks observed correspond to the redox behavior of AuNPs on the

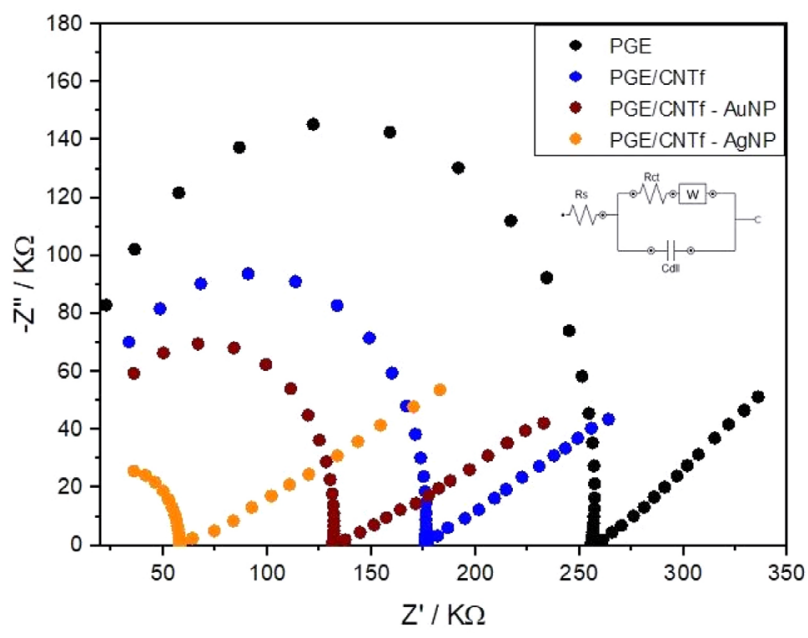

Figure 6. Nyquist diagram for the EIS measurements in $5 \mathrm{mmol} \mathrm{L}^{-1}$ $\mathrm{K}_{3} \mathrm{Fe}(\mathrm{CN})_{6} / \mathrm{K}_{4} \mathrm{Fe}(\mathrm{CN})_{6}$ and $0.1 \mathrm{~mol} \mathrm{~L}^{-1} \mathrm{KCl}$ for different electrode configurations. (inset, equivalent circuit)

electrode surface, which proves that AuNPs were effectively immobilized. ${ }^{30}$

After that, a sensor stability study was carried out to verify that the AuNPs and AgNPs immobilization process on CNTf was efficient, so that no leaching processes would occur, even when the electrode was subjected to several consecutive cycles. For this purpose, 25 consecutive cyclic voltammograms were performed on PGE/CNTf-AgNP and PGE/CNTf-AuNP, at $20 \mathrm{mV} \mathrm{s}^{-1}$ scanning rate. The voltammetric responses are shown in Figure 8.

From the voltammograms it was possible to notice that the electrochemical behavior of AgNPs and AuNPs remained the same in terms of the voltammetric profile, due to the presence of redox peaks characteristic of silver and gold, confirming the presence of the NPs with CNTf along the electrode surface after the modification process. The values of the anodic peak current (Ipa) for each case in different scanning cycles are shown in Table 2.

The results demonstrate greater stability for PGE/ CNTf-AgNP when compared to PGE/CNTf-AuNP.
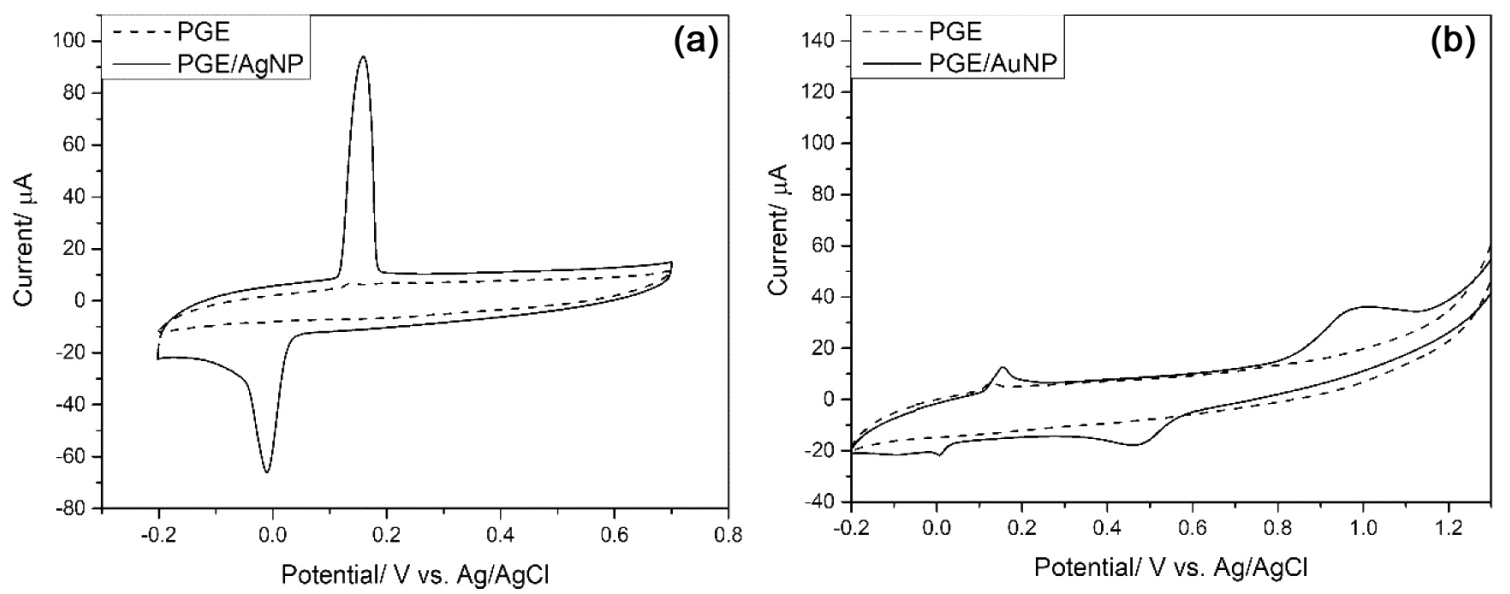

Figure 7. Cyclic voltammograms of (a) Bare PGE and PGE/ AgNP; (b) Bare PGE and PGE/AuNP. Reaction medium: $0.1 \mathrm{~mol} \mathrm{~L}^{-1} \mathrm{KCl} \mathrm{pH} \mathrm{7.0,} \mathrm{scan} \mathrm{rate} 20 \mathrm{mV} \mathrm{s}^{-1}$ 

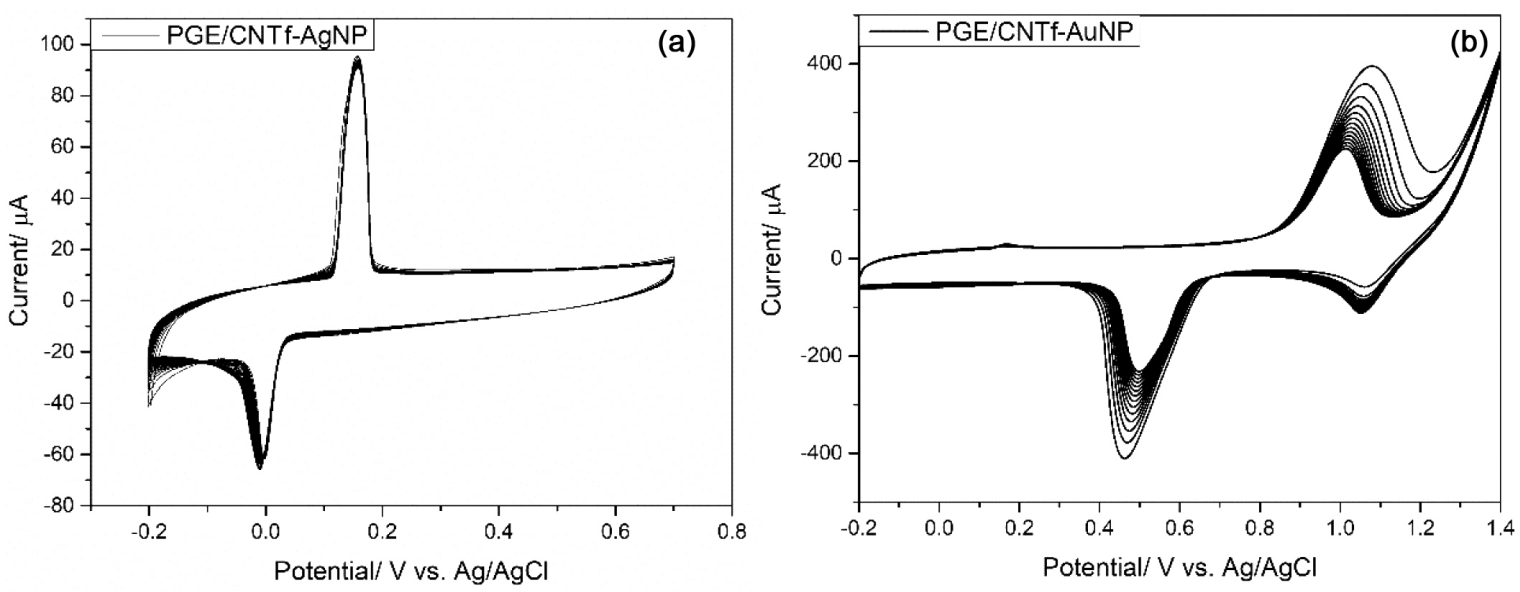

Figure 8. Several cyclic voltammograms on (a) PGE/CNTf-AgNP and (b) PGE/CNTf-AuNP. Reaction medium: $0.1 \mathrm{~mol} \mathrm{~L}^{-1} \mathrm{KCl} \mathrm{pH} \mathrm{7.0,} \mathrm{scan} \mathrm{rate} 20 \mathrm{mV} \mathrm{s}^{-1}$

Table 2. Anodic peak current (Ipa) for the PGE/CNTf-AgNP and PGE/CNTfAuNP sensors after successive cyclic voltammograms

\begin{tabular}{lcccccc}
\hline Cycle & $\mathbf{1}$ & $\mathbf{5}$ & $\mathbf{1 0}$ & $\mathbf{1 5}$ & $\mathbf{2 0}$ & $\mathbf{2 5}$ \\
\hline Ipa $(\mu \mathrm{A})$ PGE/CNTf-AgNP & 95.43 & 93.81 & 92.28 & 91.98 & 90.88 & 90.39 \\
Ipa $(\mu \mathrm{A})$ PGE/CNTf-AuNP & 395.14 & 331.67 & 288.06 & 268.71 & 252.35 & 231.72 \\
\hline
\end{tabular}

Probably, the interaction between AgNPs and CNTf was more effective than between AuNPs and CNTf, ensuring a better aggregation of the nanocomposite constituents and greater stability of the modifying layer on sensor surface. Therefore, PGE/CNTf-AgNP is the most suitable for the greatest number of analyzes, and can be subjected to several consecutive cycles without impairing the sensor efficiency.

Finally, the effective microscopic surface area was obtained by CVs for $5 \mathrm{mmol} \mathrm{L}^{-1} \mathrm{~K}_{4}\left[\mathrm{Fe}(\mathrm{CN})_{6}\right]$ in $0.1 \mathrm{~mol} \mathrm{~L}^{-1}$ $\mathrm{KCl}$ as a redox probe at various scan rates on bare PGE, PGE/CNTf, PGE/CNTf-AgNP and PGE/CNTf-AuNP. For reversible process, the the electrochemically active surface area can be estimated based on the Randles-Sevcik equation (Eq. (3))::26,28

$$
I_{p a}=2.69 \times 10^{5} n^{3 / 2} A C D^{1 / 2} v^{1 / 2}
$$

Being Ipa the anodic peak current, $n$ is the electron transfer number $(n=1)$, A the electroactive surface area of the electrode $\left(\mathrm{cm}^{2}\right)$ to be calculated, $\mathrm{C}$ the concentration of $\mathrm{K}_{4}\left[\mathrm{Fe}(\mathrm{CN})_{6}\right](\mathrm{C}=5 \mathrm{mM})$, D the diffusion coefficient $\left(\mathrm{D}=7,6 \times 10^{-6} \mathrm{~cm}^{2} \mathrm{~s}^{-1}\right.$, for $\left[\mathrm{Fe}(\mathrm{CN})_{6}\right]^{4-}$ at $\left.25^{\circ} \mathrm{C}\right)$, and $v$ the potential scan rate $\left(\mathrm{mV} \mathrm{s}^{-1}\right)$.
Thus, from a system between the Randles-Sevick equation (Eq. (3)) and the the slope of the plot of Ipa vs $v^{1 / 2}$ (Table 3), the electroactive surface areas for the different electrode configurations were calculated. The results are described in Table 3.

The results revealed that the effective microscopic surface area of PGE/CNTf-AgNP increased significantly and was about $380 \%$ greater than the microscopic area of the bare PGE being in agreement with what was expected based on the characterizations results discussed in the previous topics. Performing the modification of the base sensor with a modifying layer on the nanoscale it is expected that an increase in the sensor's surface area will occur as consequence the decrease in the average particle size of the materials. Thus, a greater percentage of atoms are available to participate in the redox reactions, contributing to the increase in the chemical reactivity and conductivity of the base sensor. Furthermore, the higher value found for the surface area of the sensor modified with the nanocomposite CNTf-AgNP, may have occurred due to the smaller average diameter obtained after the synthesis of the AgNPs, when compared to the diameter of the AuNPs.

Table 3. Values of the effective surface area from the Randles-Sevick equation and Ipa vs $v^{1 / 2}$ for different working electrode configurations

\begin{tabular}{lcc}
\hline Working electrode configurations & Ipa vs v & Surface Area $\left(\mathbf{c m}^{\mathbf{2}}\right)$ \\
\hline PGE & Ipa $=6.15 \mathrm{v}^{1 / 2}+2,50$ & 1.66 \\
PGE/CNTf & Ipa $=19.74 \mathrm{v}^{1 / 2}-69,97$ & 5.32 \\
PGE/CNTf-AuNP & Ipa $=27.52 \mathrm{v}^{1 / 2}-96,88$ & 7.42 \\
PGE/CNTf-AgNP & $\mathrm{Ipa}=29.57 \mathrm{v}^{1 / 2}-108,55$ & 7.97 \\
\hline
\end{tabular}




\subsection{Nanocomposite Effect on the Sensor Response}

Aimed to verify the modifiers effect on the working electrode reactivity, several cyclic voltammograms were recorded for the different configurations of this device (Figure 9a) in presence of $5 \mathrm{mmol} \mathrm{L}^{-1}$ of $[\mathrm{Fe}(\mathrm{CN}) 6]^{4-}$ in $0.1 \mathrm{~mol} \mathrm{~L}^{-1} \mathrm{KCl}$ solution.

The CVs exhibited the anodic and cathodic peaks referring to the reversible redox reaction of $\left[\mathrm{Fe}(\mathrm{CN})_{6}\right]^{3-14}$ for all working electrode configurations. However, in terms of Ipa, the bare PGE exhibited an extremely low Ipa, with a peak separation ( $\triangle$ Epa) of $150 \mathrm{mV}$. Moreover, the PGE/CNTf exhibited an increase in the Ipa intensity and a decrease in the peaks separation $(\Delta \mathrm{Epa}=71 \mathrm{mV})$ due to the excellent electrical conductivity of the carbon nanotubes.

The smallest peaks separation $(\Delta \mathrm{Epa}=68 \mathrm{mV})$ and the highest value of the Ipa $(60.80 \mu \mathrm{A})$ was observed to PGE/ CNTf-AgNP, indicating the high reversibility of the redox reaction and a current gain greater than $300 \%$ compared to the bare electrode (Figure 9b), which suggests that, for this configuration, the modified electrode has a much faster electronic transfer rate. The significant increase in the electrochemical response to the PGE/NTCf-AgNP can be attributed to the AgNPs catalytic effect, the synergistic effect obtained by the use of the nanocomposite and the increase in the effective surface area of the electrode resulting from a nanostructured modifying layer. ${ }^{29}$

Finally, the effect of different metallic nanoparticles on the nanocomposite formation was evaluated and compared. Thereunto, cyclic voltammograms were performed for PGE modified with CNTf-AgNP and CNTf-AuNP nanocomposites (Figure 10a). By the Ipa results, it was possible to observe that using CNTf-AgNP, there was a $50 \%$ gain in the current value (Figure 10b). This fact can be explained due to the greater electroactive surface area for this configuration, as demonstrated in the previous topic, and the greater conductivity of the silver compared to the gold conductivity.

Therefore, the use of AuNPs would only be justified if it was necessary to immobilize biological molecules on the sensor surface, due to the high biocompatibility that these nanoparticles present. In the case of applications that aimed
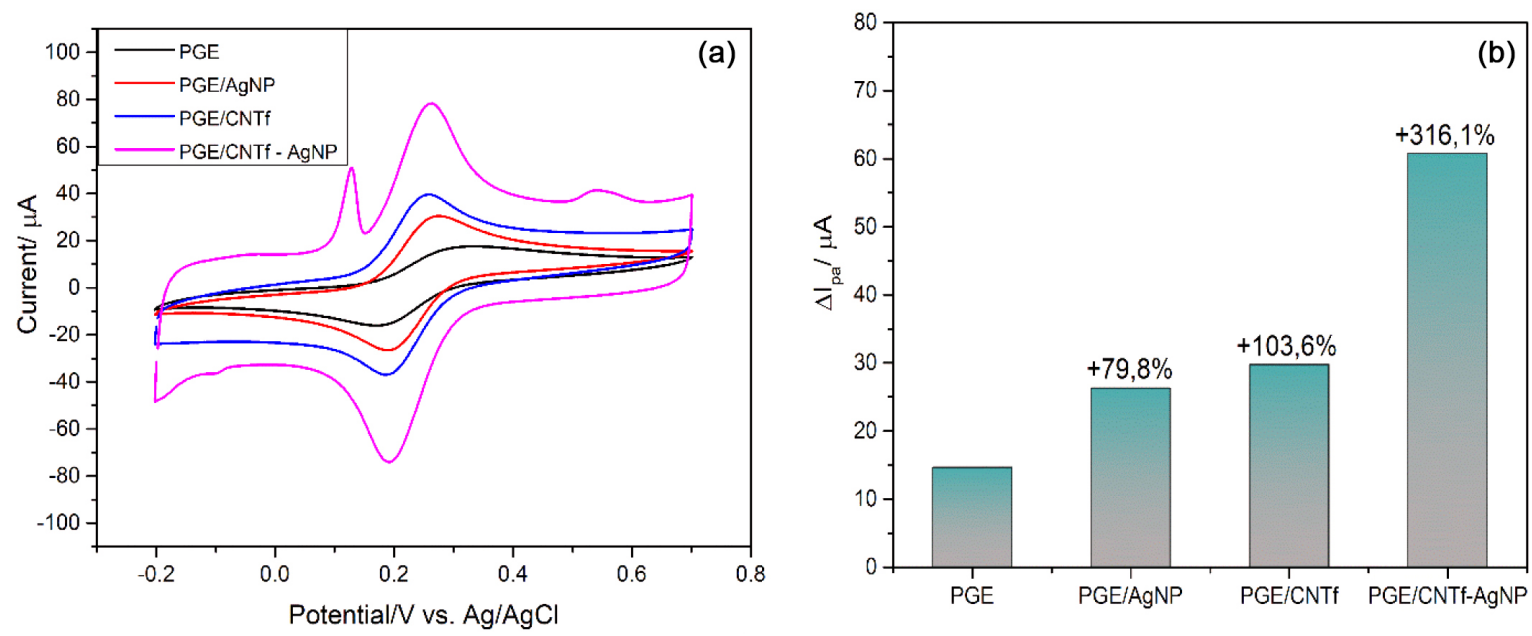

Figure 9. (a) Cyclic voltammograms referring to the different working electrode configurations; (b) Variation of the anodic peak current according to the PGE configuration. Reaction medium $5 \mathrm{mmol} \mathrm{L}^{-1}$ of $\left[\mathrm{Fe}(\mathrm{CN})_{6}\right]^{4-}$ in $0.1 \mathrm{~mol} \mathrm{~L}^{-1} \mathrm{KCl}$ solution pH 7.0
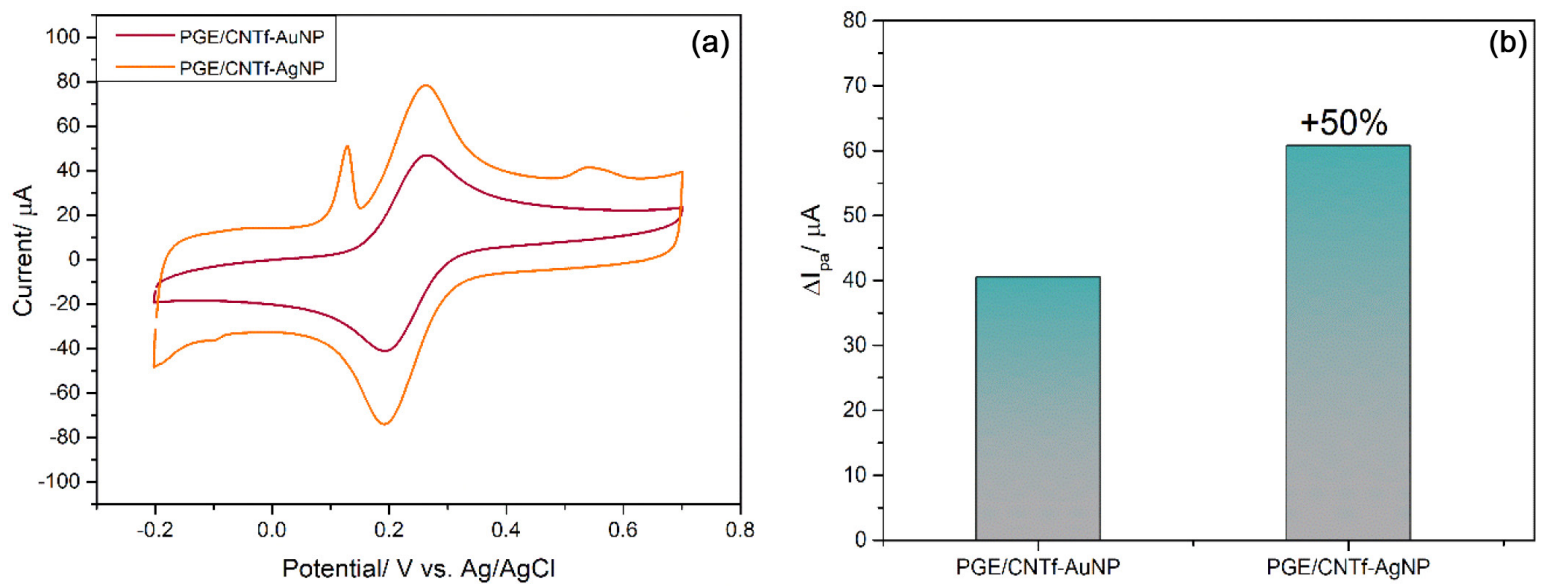

Figure 10. (a) Cyclic voltammograms referring to PGE/CNTf-AuNP and PGE/CNTf-AgNP; (b) Variation of the anodic peak current according to the PGE configuration. Reaction medium $5 \mathrm{mmol} \mathrm{L}^{-1}$ of $\left[\mathrm{Fe}(\mathrm{CN})_{6}\right]^{4-}$ in $0.1 \mathrm{~mol} \mathrm{~L}^{-1} \mathrm{KCl}$ solution pH 7.0 
fast and sensitive sensors, it is more recommendable the use of AgNPs mutually with CNTf.

\section{Conclusions}

Through the characterization stage, it was possible to confirm the presence of oxygenated functional groups in the carbon nanotubes chemical structure after treatment with strong oxidizing acids. Moreover, characterization results confirmed that homogeneous and stable colloidal dispersions of silver and gold nanoparticles were obtained.

The PGE/CNTf-AgNP and PGE/CNTf-AuNP was electrochemically characterized by EIS and $\mathrm{CV}$, confirming that the nanocomposite modified electrodes exhibited better electrochemical performance than the bare PGE. CNTf-AgNP nanocomposite shown a greater electroactive surface area, less resistance to electron transfer, considerably increasing on the electron transfer rate with a current gain $300 \%$ larger than the bare working electrode and 50\% larger than PGE/CNTf-AuNP due to the greater conductivity of the silver and better interaction and aggregation of AgNPs on CNTf, ensuring greater stability of the modifying layer on sensor surface.

Therefore, in this work, the development of a new highly sensitive voltammetric sensor prepared using functionalized carbon nanotube decorated with metal nanoparticles by an easy, fast, low coast and simple solvent evaporation method, aiming to various electrochemical applications of clinical, pharmaceutical and environmental interest, was successfully carried out offering a versatile strategy for the synthesis and assembly of other nanomaterials on CNTf with great potential for application in the development of electrochemical sensors.

\section{References}

1. Soriano, M. L.; Zougagh, M.; Valcárcel, M.; Ríos, A.; Analytical Nanoscience and Nanotechnology: Where we are and where we are heading. Talanta 2018, 177, 104. [CrossRef]

2. Goesmann, H. Feldmann, C.; Nanoparticulate functional materials. Angewandte Chemie International Edition 2010, 49, 1362. [CrossRef][PubMed]

3. Koch, C.; Structural nanocrystalline materials: an overview. Journal of Materials Science 2007, 42, 1403. [CrossRef]

4. Zhu, C.; Yang, G.; Li, H.; Du, D.; Lin, Y.; Electrochemical Sensors and Biosensors Based on Nanomaterials and Nanostructures. Analytical Chemistry 2015, 87, 230. [CrossRef] [PubMed]

5. Maduraiveeran, G.; Jin, W.; Nanomaterials based electrochemical sensor and biosensor platforms for environmental applications. Trends in Environmental Analytical Chemistry 2017, 13, 10. [CrossRef]

6. Porto, L. S.; Silva, D. N.; Oliveira, A. E. F.; Pereira, A. C.; Borges, K. B.; Carbon nanomaterials: synthesis and applications to development of electrochemical sensors in determination of drugs and compounds of clinical interest. Reviews in analytical chemistry 2019, 38, 1. [CrossRef]

7. Schroeder, V.; Savagatrup, S.; He, M.; Lin, S.; Swager, T. M.; Carbon nanotube chemical sensors. Chemical Reviews 2019, 119, 599. [CrossRef][PubMed]

8. Asadian, E.; Ghalkhani, M.; Shahrokhian, S.; Electrochemical sensing based on carbon nanoparticles: a review. Sensors and Actuators B: Chemical 2019, 293, 183. [CrossRef]

9. Shahrokhian, S.; Hafezi-kahnamouei, M.; Glassy carbon electrode modified with a nanocomposite of multi-walled carbon nanotube decorated with Ag nanoparticles for electrochemical investigation of Isoxsuprine. Journal of Electroanalytical Chemistry 2018, 825, 30. [CrossRef]

10. Branagan, D.; Breslin, C. B.; Electrochemical detection of glucose at physiological $\mathrm{pH}$ using gold nanoparticles deposited on carbon nanotubes. Sensors and Actuators B: Chemical 2019, 282, 490. [CrossRef]

11. Yusof, N. A.; Rahman, S. F. A.; Muhammad, A.; Carbon Nanotubes and Graphene for Sensor Technology. Micro and Nano Technologies 2019, 9, 205. [CrossRef]

12. Wildgoose, G. G.; Banks, C. E.; Compton, R. G.; Metal Nanoparticles and Related Materials Supported on Carbon Nanotubes: Methods and Applications. Small 2006, 2, 182. [CrossRef][PubMed]

13. Jana, N. R.; Gearheart, L.; Murphy, C. J.; Wet chemical synthesis of silver nanorods and nanowires of controllable aspect ratio. Chemical Communications 2001, 7, 617. [CrossRef]

14. Pinto, V. V.; Ferreira, M. J.; Silva, R.; Santos, H. A.; Silva, F.; Pereira, C. M.; Long time effect on the stability of silver nanoparticles in aqueous medium: effect of the synthesis and storage conditions. Colloids and Surfaces a-Physicochemical and Engineering Aspects 2010, 364, 19. [CrossRef]

15. Turkevich, J.; Stevenson, P. C.; Hillier, J.; The formation of Colloidal gold. The Journal of Physical Chemistry 1953, 57, 670. [ $\underline{\text { CrossRef] }}$

16. Haiss, W.; Thanh, N. T. K.; Aveard, J.; Fernig, D. G.; Determination of Size and Concentration of Gold Nanoparticles from UV-Vis Spectra. Analytical Chemistry 2007, 79, 4215. [CrossRef][PubMed]

17. Satyanarayana, M.; Goud, K. Y.; Reddy, K. K.; Kumar, V. S.; Gobi, K. V.; Silver nanoparticles impregnated chitosan layered carbon nanotube as sensor interface for electrochemical detection of clopidogrel in-vitro. Materials Science \& Engineering C 2019 , 101, 103. [CrossRef] [PubMed]

18. Zhang, J. Z.; Noguez, C.; Plasmonic optical properties and applications of metal nanostructures. Plasmonics 2008, 3, 127. [CrossRef]

19. Santos, J. F. L.; Santos, M. J. L.; Thesing, A. Tavares, F.; Griep, J.; Rodrigues, M. R. F.; Ressonância de plasmon de superfície localizado e aplicação em biossensores e células solares. Química Nova 2016, 39, 1098. [CrossRef]

20. Solomon, S.; Bahadory, M.; Jeyarajasingan, A.; Rutkowsky, S.; Boritz, C.; Synthesis and study of silver nanoparticles. Journal of Chemical Education 2007, 84, 322. [CrossRef] 
21. Yudianti, R.; Onggo, H.; Sudirman; Saito, Y.; Iwata, T.; Jun-ichi, A.; Analysis of Functional Group Sited on Multi-Wall Carbon Nanotube Surface. The Open Materials Science Journal 2011, 5, 242. [CrossRef]

22. Tocoglu, U.; Alaf, M.; Cevher, O.; Guler, M. O.; Akbulut, H.; The Effect of Oxidants on the Formation of Multi-Walled Carbon Nanotube Buckypaper. Journal of Nanoscience and Nanotechnology 2012, 12, 9169. [CrossRef] [PubMed]

23. Silverstein, R. M., Webster, F. X., Kiemle, D. J.; Identificação Espectrométrica de Compostos Orgânicos, 7a. ed., LTC Livros Técnicos e Científicos: Rio de Janeiro, 2006.

24. Lee, Y. J.; Park, Y.; Graphene oxide grafted gold nanoparticles and silver/silver chloride nanoparticles green-synthesized by a Portulaca oleracea extract: Assessment of catalytic activity, Colloids and Surfaces A 2020, 607, 125527. [CrossRef]

25. Lima, L. F.; Pereira, E. A.; Ferreira, M.; Electrochemical sensor for propylparaben using hybrid Layer-by-Layer films composed of gold nanoparticles, poly(ethylene imine) and nickel(II) phthalocyanine tetrasulfonate. Sensors \& Actuators: B. Chemical 2020, 310, 127893. [CrossRef]

26. Gonçalves, N. S.; Carvalho, J. A.; Lima, Z. M; Sasaki, J. M.; Size-strain study of $\mathrm{NiO}$ nanoparticles by X-ray powder diffraction line broadening. Materials Letters 2012, 72, 36. [CrossRef]
27. da Fonseca, A. F. V.; Siqueira, R. L.; Landers, R.; Ferrari, J. L.; Marana, N. L.; Sambrano, J. R.; La Porta, F. de A.; Schiavon, M. A.; A theoretical and experimental investigation of Eu-doped $\mathrm{ZnO}$ nanorods and its application on dye sensitized solar cells. Journal of Alloys and Compounds 2018, 739, 939. [CrossRef]

28. Pajooheshpour, N.; Rezaei, M.; Hajian, A.; Afkhami, A.; Sillanpää, M.; Arduini, F.; Bagheri, H.; Protein templated AuPt nanoclusters-graphene nanoribbons as a high performance sensing layer for the electrochemical determination of diazinon. Sensors \& Actuators: B. Chemical 2018, 275, 180. [CrossRef]

29. Ghalkhani, M.; Shahrokhian, S.; Ghorbani-Bidkorbeh, F.; Voltammetric studies of sumatriptan on the surface of pyrolytic graphite electrode modified with multi-walled carbon nanotubes decorated with silver nanoparticles. Talanta 2009, 80, 31. [CrossRef] [PubMed]

30. Bagheri, H.; Afkhami, A.; Khoshsafar, H.; Hajian, A.; Shahriyari, A.; Protein capped $\mathrm{Cu}$ nanoclusters-SWCNT nanocomposite as a novel candidate of high performance platform for organophosphates enzymeless biosensor. Biosensors and Bioelectronics 2017, 89, 829. [CrossRef][PubMed] 